\title{
Fabrication of the MAO coating on Mg alloy and Friction Behaviors Analysis
}

\author{
Li Changqing $^{1}$, Zhang Yunlong ${ }^{2,3 *}$, Wang Shucheng ${ }^{1}$, Zhang Yumin ${ }^{3}$, Zhou \\ Yufeng $^{3}$
}

${ }^{1}$ Colloge of Materials Sicence and Engineering, Heilongjiang University of Science and Technology, China, 150022

\author{
${ }^{2}$ College of Materials Science \& Engineering of Jiamusi University, China, 154007 \\ ${ }^{3}$ Center for Composite Materials, Harbin Institute of Technology, Harbin, China 150080 \\ allchangqing@163.com, b'ylzhdr@126.com, cwangshchlx@126.com, ${ }^{\mathrm{d}}$ zhym@ @hit.edu.cn, ${ }^{\mathrm{e}} \mathrm{zhouyf@}$ \\ hit.edu.cn
}

Keywords: MAO coating, Wear resistance, Friction rate, Mg matrix alloy

\begin{abstract}
There was a great attraction to apply magnesium alloy as a biomedical material due to its biodegradable feature and suitable mechanical properties. In order to further improve the wear resistance performance of magnesium alloy, a micro-arc oxidation (MAO) coating containing $\mathrm{MgSiO}_{3}$ was generated on the suface of $\mathrm{Mg}-\mathrm{Zn}$ alloy. The surface characterization by SEM, XRD and EDS showed that a rough and porous reaction layer with major composition of $\mathrm{O}, \mathrm{Mg}$ and $\mathrm{Si}$ was formed on the surface of Mg-Zn alloy. The result showed that the MAO coating significantly enhanced the wear resistance of $\mathrm{Mg}-\mathrm{Zn}$ alloy and friction rotate rate had not obvious on the friction coefficient of the MAO coating.
\end{abstract}

\section{Introduction}

The magnesium alloy had been well known as a new kind of biomedical material, possessing excellent mechanical properties with high strength, appropriate density and elastic modulus properties, which was similar to that of natural bones which could effectively reduce the "stress sheltering” for other metallic bone implants from the beginning of 21 century. Unfortunately, it was difficult for the rapid degradation rate of magnesium alloy to meet the requirement of rigid internal bixation, meanwhile, accompanied by the release of hydrogen gas upon degradation. So those above factors affected their clinical applications to some degree ${ }^{[1,2]}$. Surface modification technology was usually applied on magnesium and its alloys to obtain a desirable corrosion property for biomedical applications. At present, a variety of surface coating techniques had been applied on the biodegradable magnesium alloys to decrease the initial degradation rate ${ }^{[3-5]}$, which included ion implantation, chemical conversion, electrochemical deposition, anodization, micro-arc oxidation, etc. compared with above technology, the micro-arc oxidation method (MAO) could form a thick and hard coating, which possessed excellent abrasive resistance and good bonding with the substrate. In this paper, KF and EDTA-2Na were selected as additives in $\mathrm{Na}_{2} \mathrm{SiO}_{3}-\mathrm{NaOH}$ solution to prepare the ceramic coatings by micro-arc oxidation technology. MAO coatings containing $\mathrm{MgSiO}_{3}$ phase were fabricates on the surface of the magnesium alloys and microstructure was analysized. The effect of the friction rotating rate on the the friction behaviors were investigated in details.

\section{Materials fabrication and characterization}

The detailed preparation procedure of the MAO coating was describedas follows.

The hot extrusion Mg-Zn alloy was utilized as substrate materials. Before the MAO coatings, the specimens were cut in the sizes of $10 \mathrm{~mm} \times 10 \mathrm{~mm} \times 5 \mathrm{~mm}$. Surfaces of the investigatived $\mathrm{Mg}$ alloy specimens were ground to grids of 600,1200 and 2000 by SiC papers and cleaned in distilled water and acetone. Then a silicate containing electrolyte $\left(16 \mathrm{~g} / \mathrm{L} \mathrm{Na}{ }_{2} \mathrm{SiO}_{3}, 6 \mathrm{~g} / \mathrm{L} \mathrm{NaOH}\right.$, and $\left.4 \mathrm{~g} / \mathrm{L} \mathrm{KF}\right)$ was 
adopted for the MAO treatment. The alloy specimen was taken as the working electrode and the counter electrode was the stainless steel container. The voltage, work frequency, duty cycle and preparation time were $320 \mathrm{~V}, 1000 \mathrm{~Hz}, 20 \%$ and $10 \mathrm{~min}$, respectively. After MAO treatment, the samples were soaked thoroughly in distilled water and dried in air. The wear properties of the composites was measured by SFT-2M pin on disk wear tester. The abrasion counterpart was made of steel of GCr15 and rotating radius was $3 \mathrm{~mm}$ and load was $300 \mathrm{~g}$. The rotating rate was different as 300rpm, 600rpm, 900rpm and 1200rpm, which were nominated as R300, R600, R900 and R1200. The phase composition was analyzed using X-ray diffractometer (XRD, D/max2500) with $\mathrm{Cu} \mathrm{K \alpha}$ radiation. The microstructure of the MAO coating was observed on a scanning electron microscopy (HITACHI/S-3700). The chemical composition of the coating was determined by the energy dispersive spectrum (EDS, Oxford 300) analysis.

\section{Results and discussion}

The XRD pattern of MAO coating of the Mg-Zn alloy were illustrated in Fig.1. In the matrix specimen of the Mg-Zn alloy, the Mg phase was considered as the main phase, the main diffraction peak was consistent with Mg (No.65-3365\#). While after MAO process, the MgO (No.45-0946\#) and $\mathrm{MgSiO}_{3}$ (No.19-0768\#) phase was detected as the second trace phase, meanwhile the diffraction peak of $\mathrm{Mg}$ phase also can be observed. Based on the above results, we can draw to a conclusion that the $\mathrm{MgO}$ and $\mathrm{MgSiO}_{3}$ phase can be formed during the $\mathrm{MAO}$ process.

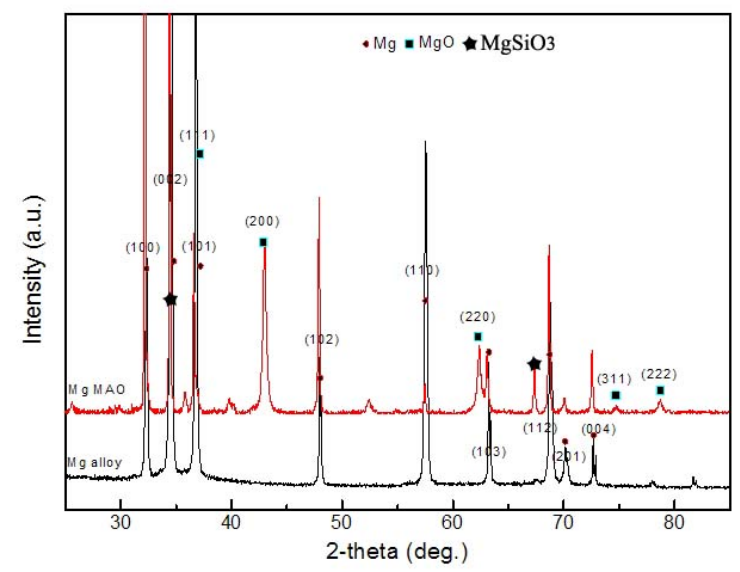

Fig.1 XRD pattern of MAO oxidation coating of the Mg-Zn alloy

The surface morphology and energy spectrum of MAO coating of the Mg-Zn alloywas illustrated in Fig.2. Fig.2(a) and (b) represented high magnification image and energy spectrum of spot “+” in Fig.2a). According to the Fig.2, the pore was uniform and the surface was smooth. It was interesting that micro cracks with appreciable quantity can not be observed. It was noted that more micro cracks can be found from the MAO coating from the previous reports. From this viewpoint, the application of appreciable surface modifier could decorate micro-structure of MAO coating, so it played an important role on the friction properties of the Mg-Al-Y alloy. The energy spectrum of spot "+" in inset of Fig.2(b) was analysed and Mg, Si and O element can be detected. The $\mathrm{O}$ and $\mathrm{Si}$ element were introduced into the oxide coating surface of the Mg-Zn alloy by the MAO methods.

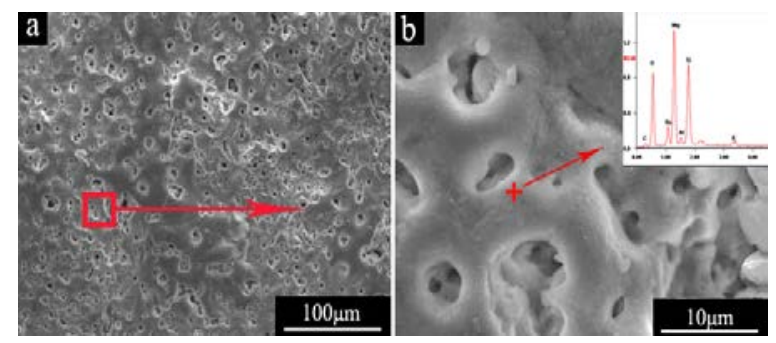

Fig.2 The surface morphology and energy spectrum of the MAO coating specimen 
Fig.3 showed worn surface morphology of the MAO coating composites. a),b),c) and d) represent the worn surface of MAO coating under the different friction rate as 300rpm, 600rpm, 900rpm and 1200rpm. It can be clearly seen that the traces of worn specimen was deeper gradually with increase of friction rate. As the friction rate was about 300rpm, the scratch was shallow and the degree of the surface damage was low. A small number of cracks can be found from inset of the Fig.4. As friction rate was beyond 900rpm, the degree of the surface damage was strengthened. The typical Grooved scratches can be detected from surface morphology of the MAO coating. From the data, it can be judged that $t$ adhesive wear was the main worn mechanism. The wearing resistance of the MAO coating composites could be enhanced after the MAO process compared with the Mg matrix materials.

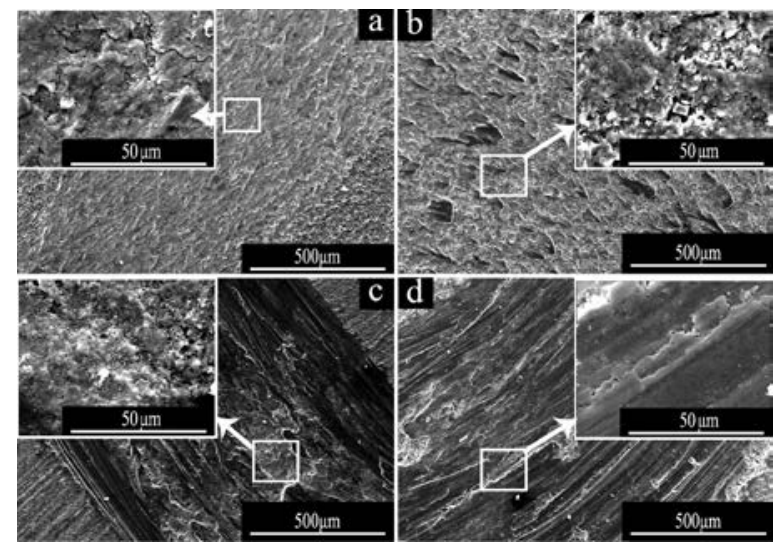

Fig. 3 the worn surface morphology of the MAO coating composites.

Fig.4 showed the relationship curve between friction coefficient and friction rate under different sliding time for the Mg-MAO coating. It was clear that the friction coefficient of measured sp ecimens varied in the range of $0.40 \sim 0.65$, which was accompanied by severe oscillation. At the beginning of the friction process, the friction condition of the MAO coating was kept in the unstable, which procedure had correlations with the densification state of the surface loosen layer of MAO coating. As we all known, the MAO coating was composed of loosen layer, transition layer and densification layer. When the loosen layer was damaged, the friction process became stable. As the friction rate enhangced, the volatility amplitude became stronger. So we can draw to a conclusion that the friction rotate rate had not obvious on the friction coefficient.

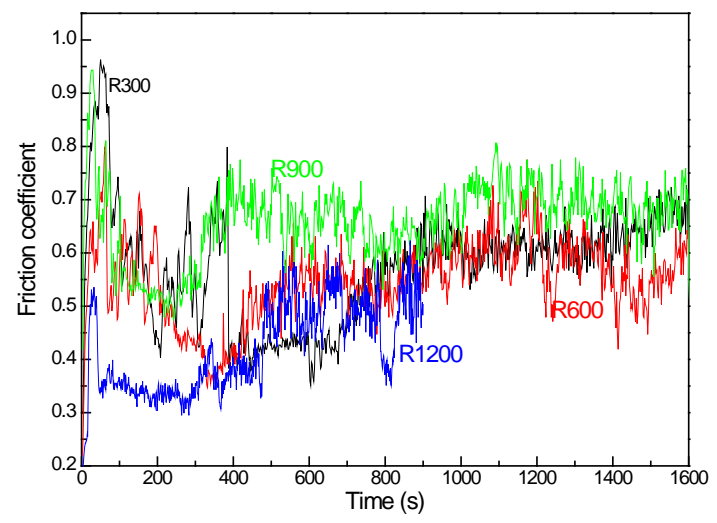

Fig.4 The relationship curve between friction coefficient and friction rate of the MAO coating

\section{Conclusions}

The ceramic coatings were formed on $\mathrm{Mg}-\mathrm{Zn}$ substrate by $\mathrm{MAO}$ method in $\mathrm{Na}_{2} \mathrm{SiO}_{3}-\mathrm{NaOH}$ solution and the conclusions could be gained as follows. A micro-arc oxidation (MAO) coating containing $\mathrm{MgSiO}_{3}$ was generated on the suface of $\mathrm{Mg}-\mathrm{Zn}$ alloy in order to further improve the wear resistance performance of magnesium alloy. The surface characterization by SEM, XRD and EDS showed that a rough and porous reaction layer with major composition of O, Mg and Si was formed on the surface of Mg-Zn alloy. The result showed that the MAO coating significantly 
enhanced the wear resistance of Mg-Zn alloy and friction rotate rate had not obvious on the friction coefficient of the MAO coating.

\section{Acknowledgments}

The authors were grateful for the support by the science and technology research project of education department of Heilongjiang Province (No.12531586). The Major State Basic Research Development Program (No.2014CB46505) and The science and technology innovation team of Jiamusi university(No.Cxtd-2013-03).

\section{References}

[1]B. Jiang, D.F. Zhang, J. Peng, et al. Application and investigation of Mg-Li alloys, Mater. Rev. 2005 (19):38-41.

[2] Hongfei Guo, Maozhong An, Shen Xu, et al. Microarc oxidation of corrosion resistant ceramic coating on a magnesium alloy, Mater. Lett. 2006(60): 1538-1541.

[3] Zhongping Yao, Huihui Gao, Zhaohua Jiang, et al., Structure and properties of $\mathrm{ZrO}_{2}$ ceramic coatings on AZ91D Mg alloy by plasma electrolytic oxidation, J. Am. Ceram. Soc. 2008(91): 555-558.

[4] H.P. Duan, C.W. Yan, F.H. Wang. Growth process of plasma electrolytic oxidation films formed on magnesium alloy AZ91D in silicate solution, Electrochim. Acta, 2007(52):5002-5009.

[5]H.F. Guo, M.Z. An, Effect of surfactants on surface morphology of ceramic coatings fabricated on magnesium alloys by micro-arc oxidation, Thin Solid Films. 2006(500): 186-189. 